\title{
Random and channeled energy loss in thin germanium and silicon crystals for positive and negative 2-15-GeV/c pions, kaons, and protons
}

\section{Citation}

Esbensen, H., O. Fich, J. A. Golovchenko, S. Madsen, H. Nielsen, H. E. Schiøtt, E. Uggerhøj, et al. 1978. Random and Channeled Energy Loss in Thin Germanium and Silicon Crystals for Positive and Negative 2-15-GeV/c Pions, Kaons, and Protons. Physical Review B 18, no. 3: 18-1054 . doi:10.1103/physrevb.18.1039.

\section{Published Version}

doi:10.1103/PhysRevB.18.1039

\section{Permanent link}

http://nrs.harvard.edu/urn-3:HUL.InstRepos:29407064

\section{Terms of Use}

This article was downloaded from Harvard University's DASH repository, and is made available under the terms and conditions applicable to Other Posted Material, as set forth at http:// nrs.harvard.edu/urn-3:HUL.InstRepos:dash.current.terms-of-use\#LAA

\section{Share Your Story}

The Harvard community has made this article openly available.

Please share how this access benefits you. Submit a story.

Accessibility 


\title{
Random and channeled energy loss in thin germanium and silicon crystals for positive and negative $2-15-G e V / c$ pions, kaons, and protons
}

\author{
H. Esbensen, O. Fich, J. A. Golovchenko*, S. Madsen, H. Nielsen, H. E. Schiøtt, \\ E. Uggerh $\varnothing \mathrm{j}$, and C. Vraast-Thomsen \\ Institute of Physics, University of Aarhus, DK-8000 Aarhus C., Denmark
}

G. Charpak, S. Majewski, G. Odyniec, G. Petersen, and F. Sauli CERN, CH-1211 Geneve 23, Switzerland

J. P. Ponpon and P. Siffert

Centre de Recherches Nucléaires, PHASE, F-67037 Strasbourg-Cedex, France

(Received 12 December 1977)

\begin{abstract}
Energy loss of 2-, 6-, and $15-\mathrm{GeV} / c$ positive and negative protons, kaons, and pions transmitted through thin germanium and silicon crystals are measured using the crystals as intrinsic detectors. For particles incident in directions far from axial or planar directions the results are in accord with the relativistic BetheBloch stopping theory. The density effect plays an important role at these energies. The spectral distribution of energy losses is in good agreement with the Landau distribution. For positive particles aligned with an axis or a plane, the energy loss is considerably reduced compared to random. The measurments agree well with theoretical calculations. Negative particles, well aligned to a crystal axis, show increased energy loss compared to random by as much as $\sim 20 \%$.
\end{abstract}

\section{INTRODUCTION}

In a previous experiment directional effects were observed for charged heavy particles with energies around $1 \mathrm{GeV}$ penetrating through germanium single crystals. The probability of both small- and largeangle scattering depended strongly on the relative orientation of the crystal and the particle beam, with positive and negative particles behaving differently. Also the energy-loss spectra were highly influenced by crystal orientation. The directional, or channeling, effects appear when the angle between the beam and a crystal axis or plane is smaller than a certain critical angle, which, for energies around $1 \mathrm{GeV}$, is of the order of $1 \mathrm{mrad}$. In the channeling case, collisions with atoms become correlated so that all deflections with a string or plane of atoms tend to be in the same direction. A positive particle is therefore gently steered away from the atomic string or plane. This is the reason why channeling effects appear: channeled particles are kept away from the close vicinity of atoms.

Preliminary results of the experiment were published in Refs. 1 and 2, and a fuller discussion of the results together with a summary of the theoretical background is presented in Ref. 3 . Thus having solved various problems associated with experimentally observing channeling effects at energies as high as $1 \mathrm{GeV}$, it has become possible for us to pursue the effects at even higher energies. We have been motivated to continue the study of the channeling effect first of all because of its basic physical interest but also because of its possible implications for high-energy physics experiments.

A new experiment has now been performed, using particles with momenta 2,6 , and $15 \mathrm{GeV} / c$ and various germanium and silicon crystals. The present paper deals with one aspect of the results from the new experiment, namely, the measurements of energy loss in the single crystals. In addition to the energy loss under channeling conditions, it is possible to measure the corresponding energy loss for an amorphous target since (cf. Ref. 3) the interaction between an energetic particle and a single crystal is the same as that for an amorphous foil whenever the direction of motion of the particle is far from any axial or planar direction in the single crystal. Such directions are called random directions; they give rise to random energy loss. Another interesting feature of these results is that at the present energies the well-known relativistic Bethe-Bloch ${ }^{4}$ formula for the average energy loss of random particles is strongly modified by the so-called density effect. Our measurements on the energy loss of random particles provide experimental support to Sternheimer's calculations ${ }^{5}$ of the density effect.

For well-channeled positive particles there exist many theoretical papers predicting the energy loss of the particles. The theories are conflicting and none of them are readily applicable at extreme relativistic energies. We present a com- 
prehensive tabulation of energy-loss data for pions, kaons, and protons, channeled along axial and planar directions in silicon and germanium for momenta of 2,6 , and $15 \mathrm{GeV} / c$. We find good agreement between the data and a recent theory of Esbensen and Golovchenko. ${ }^{6}$

A theoretical description of the energy loss for negative channeled particles is not straightforward. Qualitative arguments lead to the expectation that channeled negative particles should experience a larger than random energy loss. Until lately electrons were the only negative particles to be used in channeling; due to several experimental problems (dechanneling, bremsstrahling, etc.), energy-loss experiments with well-aligned electrons are not very attractive, and only one has been performed to our knowledge. ${ }^{20}$

In the old experiment no appreciable difference between a channeled-and a random-energy-loss spectrum was observed for negative pions around $1 \mathrm{GeV} / c$. In the present experiment, however, with improved angular resolution we have found a considerable shift of the energy-loss spectrum compared to the random-energy-loss spectrum. Measurements on the interesting scattering effects of channeled particles as well as applications to nuclear reactions $w$ ill be published separately.

\section{EXPERIMENTAL SETUP}

\section{A. General layout}

The experimental apparatus was installed in the P14 beam of the CERN $28-\mathrm{GeV} / c$ proton synchrotron. A schematic layout of the experiment can be seen in Fig. 1. The beam itself was a high-intensity (around $10^{6} / \mathrm{cm}^{2}$ sec.) secondary nonseparated charged beam with a momentum adjustable between 2 and $15 \mathrm{GeV} / c$. Momentum slits allowed a reduction of intensity to acceptable limits for the detectors. In the positive polarity the beam consisted of roughly equal amounts of protons and pions with about $2 \%$ of kaons, whereas in the negative polarity it contained essentially only pions with $1 \%$ of antiprotons and kaons. The beam divergence was related to the momentum as shown in Table I.

Particle identification was performed in two
TABLE I. Beam divergence for both polarities and for the momenta used in the experiment. The numbers give full width at half-maximum in the horizontal and vertical directions in milliradians.

\begin{tabular}{clcc}
\hline $\begin{array}{c}\text { Momentum } \\
(\mathrm{GeV} / c)\end{array}$ & Polarity & Horizontal & Vertical \\
\hline 15 & positive & 0.7 & 0.3 \\
15 & negative & 1.7 & 1.3 \\
6 & positive & 1.4 & 0.8 \\
6 & negative & 1.6 & 1.6 \\
2 & positive & 1.8 & 1.4 \\
2 & negative & 3.2 & 2.5 \\
\hline
\end{tabular}

ways: For 2-GeV/c particles the time of flight between the two scintillation counters SC1 and SC4 $16 \mathrm{~m}$ apart was recorded. For the higher momenta two threshold Čerenkov counters placed in front of SC1 were employed. The gas pressure in the Čerenkov counters was chosen so that a pion would give a signal in both, a kaon a signal in one and not in the other, and a proton would give no signal in either of the two detectors. Two additional scintillation counters SC2 and SC3 in anticoincidence with SC1 and SC4 were used to define the usable fraction of the beam so as not to exceed the maximum size of the crystal sample at the focal point on the goniometer.

The dominant contribution to the angular uncertainty in the old experiment came from multiple Coulomb scattering of the particles in air gaps and windows. Multiple scattering decreases as $1 / p v$, whereas the critical angle for channeling decreases as $1 \sqrt{p v}$, where $p$ and $v$ are the relativistic momentum and the velocity of the particle, respectively. The angular resolution may therefore be improved by going to higher energies. In addition, a special effort was made to design the experiment so as to further reduce the multiple scattering.

The incoming as well as the outgoing particle trajectories were measured by a set of five positionsensitive drift chambers, DC1-DC5; the beam lines VT1 and VT2 were evacuated to a pressure of less than $10^{-2}$ Torr which essentially eliminates the contribution to multiple scattering from the air in the tubes. The goniometer allowed an adjustment

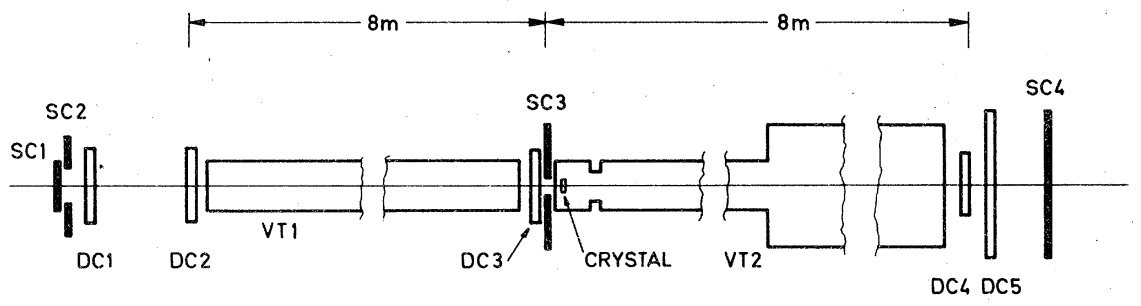

FIG. 1. Schematic drawing of experimental layout. The beam is defined by means of scintillation counters SC 1-4, and the particle track by means of drift chambers DC 1-5. VT1 and VT2 are evacuated tubes. 
of the crystal axis to the average beam direction, although a precise alignment was not necessary due to our method of measurement. A suitable liquid-nitrogen cooling system could maintain the intrinsic detector at a nearly constant temperature of around $90^{\circ} \mathrm{K}$ and the pressure was kept below $10^{-6}$ Torr.

\section{B. Detecting system}

The basic principle of the experiment was to record, for each beam particle obeying the simple geometrical constraints already described, both the incoming and the outgoing angle to the crystal and at the same time to register the energy deposited in the crystal. For this purpose we have used a set of high-accuracy drift chambers to record on each event, the two-dimensional $x-y$ coordinates of the tracks on the selected planes (DC1-DC5 in Fig. 1). The active surface was $100 \times 100 \mathrm{~mm}$ for DC1-DC4, whereas DC5 was $500 \times 500 \mathrm{~mm}$. DC5 was used for recording large scattering angles. The detailed behavior of drift chambers has been described elsewhere. ${ }^{3}$

The angular resolution of the detecting system is influenced by two causes: Firstly, there is an uncertainty in the coordinates from the drift chambers and the associated electronics of $\sim 0.1 \mathrm{~mm}$ corresponding to a total angular uncertainty of $\sim 0.03 \mathrm{mrad}$. Secondly, there is an uncertainty in the particle directions when entering and leaving the crystal because of multiple scattering in both the mylar windows and the airgaps close to the crystal. This second effect is more serious at the lower energies, and in the actual setup is much worse on the incident side than on the exit side. The multiple scattering contribution was calculated in the same way as in Ref. 3, i.e., based on the Bohr-Williams theory. The calculated angular resolution (standard deviation) of incident and exit beams are tabulated in Table II as well as the corresponding total angular resolution through the system. These numbers are in good accord with measurements performed on the system without the crystal in place.

As mentioned above, the critical angles for channeling are very small at these high energies.

TABLE II. Standard deviation in measurement of incident and exit beam angles (mrad) as well as the total standard deviation.

\begin{tabular}{lccc}
\hline & $2 \mathrm{GeV} / c$ & $6 \mathrm{GeV} / c$ & $15 \mathrm{GeV} / c$ \\
\hline$\sigma_{\text {incident }}$ & 0.55 & 0.19 & 0.08 \\
$\sigma_{\text {exit }}$ & 0.06 & 0.04 & 0.03 \\
$\sigma_{\text {total }}$ & 0.55 & 0.19 & 0.09 \\
\hline
\end{tabular}

TABLE III. The critical angle $\psi_{1}$ in mrad. The tabulated values are for $\beta=1$.

\begin{tabular}{lccc}
\hline & $2 \mathrm{GeV} / c$ & $6 \mathrm{GeV} / c$ & $15 \mathrm{GeV} / c$ \\
\hline $\mathrm{Si}\langle 110\rangle$ & 0.32 & 0.19 & 0.12 \\
$\mathrm{Ge}\langle 110\rangle$ & 0.48 & 0.28 & 0.18 \\
\hline \hline
\end{tabular}

For relativistic particles, the critical angle for axial channeling $\psi_{1}$ is given in the Lindhard theory ${ }^{7}$ by

$$
\psi_{1}=\left(4 Z_{1} Z_{2} e^{2} / p v d\right)^{1 / 2},
$$

where $Z_{1} e$ and $Z_{2} e$ are the nuclear charges of the incident particle and the target nuclei, respective$\mathrm{ly}$, and $d$ is the interatomic distance along the axis. This theoretical critical angle has turned out to be in.good agreement with experiments. In Table III values of $\psi_{1}$ for the present experiment are given. We have chosen a $\langle 110\rangle$ axis, for which $d=4.00 \AA$ in $\mathrm{Ge}$, and $d=3.84 \AA$ in Si. Critical angles for planes are of the order of $0.3 \psi_{1}$ at these energies. It is apparent that a good angular resolution is necessary in order to accurately measure the channeling effects.

The data acquisition and handling were in principle done as described in Ref. 3. In short, for each accepted event, the output from the drift chambers, from the crystal detector, and from the Cerenkov counters, viz., the time-of-flight measurement, were stored on magnetic tape. The full analysis of the data was done on the CDC 7600 at CERN, while a small on-line computer checked the correct behavior of the detecting system.

\section{Preparation of crystal detectors}

Few elements form crystals which are suited for high-energy channeling experiments, since the critical angles are so small that even a moderate mosaic spread will smear the channeling effects. In all experiments we have used silicon and germanium crystals which can be produced with very low mosaic spread. These materials are also suitable for the fabrication of solid-state ionization detectors as discussed below.

A slight bending of the crystals will also lead to a smearing of the effects and in order to avoid this, we had to construct special crystal holders where the crystals were held fixed without any applied stress. Crystals mounted this way were tested at room temperature by means of a double crystal $x$ - ray spectrometer. From the rocking curve a very accurate measurement of the bending was obtained. Also $x$-ray topography was employed. By these means it was ensured that the crystals were bent no more than $10^{-2}$ mrad. 
When running the experiment, we did a final test on the crystal bending by means of the transmission effect (see Sec. III A). It should be noted that in the absence of the above-mentioned precautions, it was not uncommon to encounter mounted samples bent by as much as $5 \mathrm{mrad}$.

In order to use the crystals as pulse-height detectors, they were prepared in the following way: germanium crystals were treated as reported in Ref. 3. In short, high-purity $n$-type material was used with a net impurity concentration below 2.5 $\times 10^{10} / \mathrm{cm}^{3}$. Samples of various thicknesses ranging from 0.3 to $4.2 \mathrm{~mm}$, and with a $\langle 110\rangle$ axis perpendicular to the surface, were prepared. Low-energy boron implantation was used without any further annealing to realize the front rectifying contact and a 200- $\check{A}$ aluminum layer was deposited as a back contact. This procedure, where there is no heating of the sample, ensures that the bulk of the material maintains its quality. These samples need to be cooled only during experiments.

Silicon crystals were made of $n$-type material with resistivity in the range 10000-30000 $\Omega \mathrm{cm}$. Samples of thickness between 0.3 and $1 \mathrm{~mm}$, and with $a\langle 110\rangle$ or a $\langle 111\rangle$ axis perpendicular to the surface were prepared. The front contact was made by implantation of $10^{14} / \mathrm{cm}^{2} 5-\mathrm{keV}$ boron ions followed by a $30-\mathrm{min}$ annealing at $200{ }^{\circ} \mathrm{C}$ in order to remove the radiation damage. We used the same back contact as for the germanium crystal.

Thus the crystals in which the channeling conditions are established during the experiment are also solid state ionization detectors. When a suitable bias voltage is applied to these crystals there will be an output pulse from the associated electronics, which is accurately proportional to the energy deposited in the crystal by ionization processes. Energy calibration of the system is determined by irradiating the crystals with radioactive cobalt and barium sources. In most cases the detectors were fully depleted during the experiments, but the effective sensitive thickness could be varied by varying the bias.

\section{Crystal alignment}

As mentioned above, the incident beam is not parallel and so it is only necessary to align the crystal axis within the cone of incoming angles. In the previous experiment the transmission patterns were utilized to establish the direction of the crystal planes. For each setting of the goniometer, a magnetic tape was filled and analyzed, and corrections in the crystal setting were then made. This process was repeated until perfect alignment was obtained. It was rather time consuming, es-

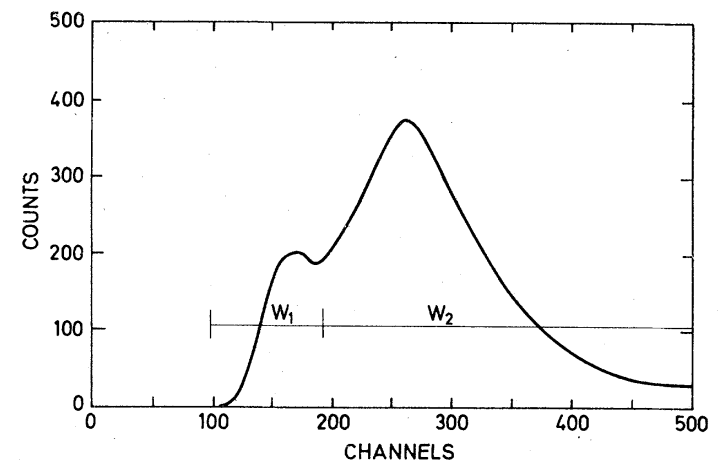

FIG. 2. Multichannel analyzer spectrum from the solid-state detector. The low-energy part comes from channeled particles, whereas the high-energy part is caused by "random" particles. The windows W1 and W2 are set in order to align the crystal to the beam.

pecially since the crystal axis could be off the incident beam cone by as much as a couple of degrees.

In the present case the alignment was performed in a different and much faster way. We used the fact that positive particles experience lower than random energy loss when channeled along planes or axes. The pulse-height spectrum from the crystal for all particles was fed into a multichannel analyzer. Such a spectrum is shown in Fig. 2. Actually, this spectrum shows a situation where the alignment is already perfect. The low-energyloss group corresponds to the channeled particles. In a first setting of the crystal only a small part of the beam particles happen to be aligned to some crystal planes, and the low-energy-loss part of the spectrum is only a weak tail.

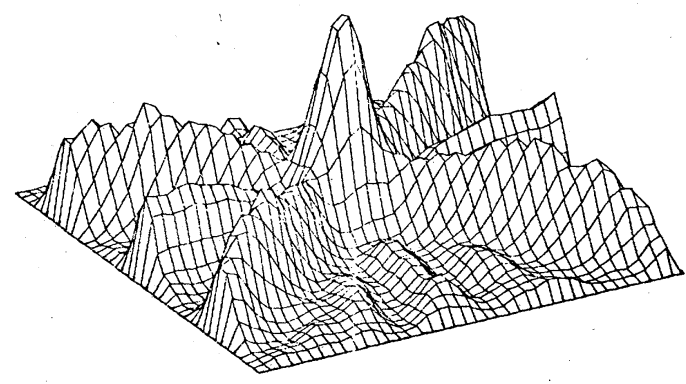

FIG. 3. 6-GeV/c $\pi^{+}$incident on a $0.3-\mathrm{mm}$ Ge single crystal. The picture shows the relative intensity distribution as function of incident angle to the crystal axis for particles belonging to the low-energy-loss window W1. The distance between lines in $x$ and $y$ directions is $0.05 \mathrm{mrad}$. There is a strong peak in the string direction as well as pronounced crests in the planar directions. There are two strong $\{111\}$ planes, with a $\{110\}$ plane in between. Weaker planes are also visible. 
Electronically, two windows are set, one (W1) covering the low-energy-loss tail, the other (W2) covering the rest of the spectrum. W2 is used for normalization. When the relative number of particles in $\mathrm{W} 1$ is plotted against crystal tilt angle, a peak is recorded each time a plane is aligned to the beam. In this way planes and axis are found very quickly without any use of a computer and with a goniometer with a minimum step size of $\sim 0.2$ mrad.

In Fig. 3 is shown a three-dimensional intensity plot for $6 \mathrm{GeV} / c \pi^{+}$incident on a $0.3-\mathrm{mm}$ Ge crystal with a $\langle 110\rangle$ axis aligned to the beam. The particles registered belong to the low-energy-loss window W1. Even weak planes are detectable this way.

\section{RESULTS AND DISCUSSION}

\section{A. Transmission experiment}

We can further elucidate the channeling picture as well as supplement the information of Fig. 3 by means of a transmission experiment. Here one asks for the direction of incidence of those particles which traverse the crystal without essential deflection. Figure 4 shows the intensity distribution in angle space of $15-\mathrm{GeV} / c$ protons transmitted through a $4.2-\mathrm{mm}\langle 110\rangle$ Ge crystal. The maximum accepted angle between incident and exit directions was $0.1 \mathrm{mrad}$. It is seen that incident protons aligned with the $\langle 110\rangle$ axis or the lower-order planes give rise to a strongly increased transmission intensity, whereas a reduction in transmis-

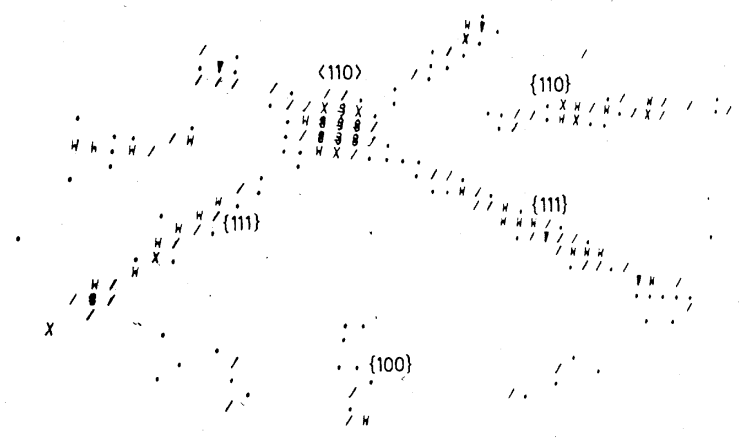

FIG. 4. $15-\mathrm{GeV} / c p$ incident on a $4.2-\mathrm{mm}$ Ge single crystal. The picture is a reproduction of a computer output and shows the relative intensity distribution as function of incident angle to the crystal axis for particles which have been scattered less than $0.1 \mathrm{mrad}$ in the crystal. Dark areas correspond to high intensity. Each point shows the intensity in a box of $0.05 \times 0.05 \mathrm{mrad}$. As in Fig. 3 the position of the axis and the planes are clearly seen. Note the reduction in yield away from axis and planes for incident angles less than around $1 \mathrm{mrad}$. There are 20000 events in the plot.

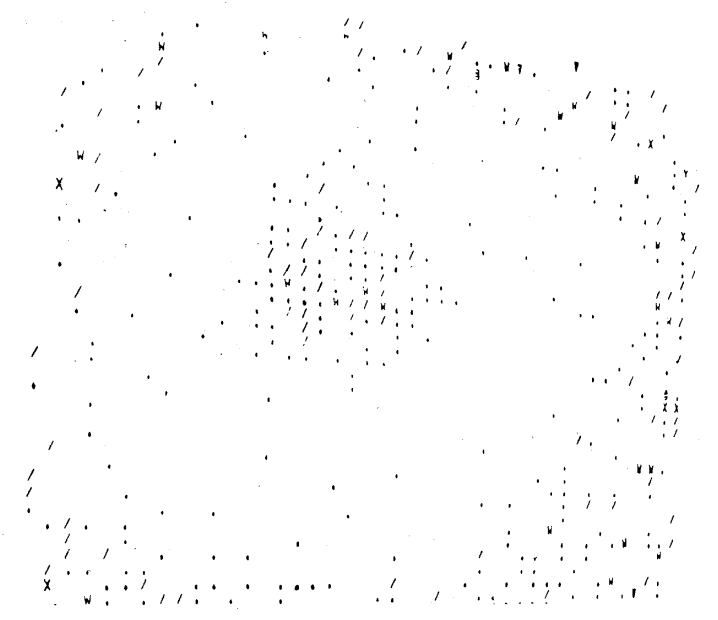

FIG. 5. $15-\mathrm{GeV} / c \pi^{-}$incident on a $4.2-\mathrm{mm}$ Ge single crystal. Same plot and condition as in Fig. 4 but for negative particles. Here planar effects are absent but there is a peak in the axial direction surrounded by a low-intensity region. Note that the axial peak is lower than the randon "plateau" far from the axis. There are 10000 events in the plot.

sion yield is observed away from axis and planes for incident angles up to around $1 \mathrm{mrad}$. The minimum in transmission intensity is obtained at an angle of around $0.3 \mathrm{mrad}$ to the axis.

Figures 3 and 4 make it easy to select special particle groups, as, for example, all particles aligned to a certain plane, or those particles which make a specified angle to the axis, etc., and to ask what happens to such a particle group as regards scattering angles, energy loss, etc. The transmission pattern was also used as a last test of the possible bending of the crystal. By means of the computer, the pattern from various parts of the crystal is obtained. If the angular position of the axis differs from one part of the crystal to the next, the crystal is bent, and the angle of bending is immediately found to an accuracy of around 0.02 mrad.

For negative particles the transmission picture looks a little different. Figure 5 shows the intensity distribution of $15-\mathrm{GeV} / c \pi^{-}$transmitted through the same crystal as above. There is still a peak along the $\langle 110\rangle$ axis, but the intensity is lower $(\sim 30 \%)$ than the random intensity. Again the peak is surrounded by a low-intensity region, which in the present case extends out to around $2 \mathrm{mrad}$ as compared to $1 \mathrm{mrad}$ for protons. The intensity minimum is found at nearly the same angle as for protons. No clear planar effects are seen.

The reason for the low-intensity region is one of the main topics of a subsequent paper. In short, particles (positive or negative) entering the crystal 
with an angle to the axis (polar angle) up to around the critical angle, and some azimuthal angle, will be scattered by the atomic string in such a way that the polar angle is conserved (conservation of transverse energy) whereas the azimuthal angle changes. Within some $10-20 \mu$ of penetration depth the distribution in angle space becomes ring shaped, the radius of the ring being equal to the polar angle. The small scattering angle criterion applied for transmission experiments excludes most of the particles in the ring, hence giving rise to the low-intensity region.

\section{B. Energy-loss experiment \\ 1. Positive particles}

We now present some typical pulse-height spectra from the silicon and germanium crystal detectors. The energy scale was obtained as mentioned above. Note that the spectra display the distribution of energy deposited in the depletion layers of the crystal. Although, as discussed below, this quantity is slightly less than the energy loss of the particle we shall in the following continue to use the phrase energy loss. In each figure two spectra are shown, an aligned spectrum (along the axis or some plane) for which strict angular criteria are set on both the incident and exit beam, and a random spectrum obtained with the crystal tilted so that the axis and planes are far outside the beam cone.

The results for $15-\mathrm{GeV} / c$ protons incident on a 740- $\mu$ Ge crystal are displayed in Figs. 6-9. The aligned spectra correspond to channeling along the $\langle 110\rangle$ axis, and the $\{111\},\{110\}$, and $\{100\}$

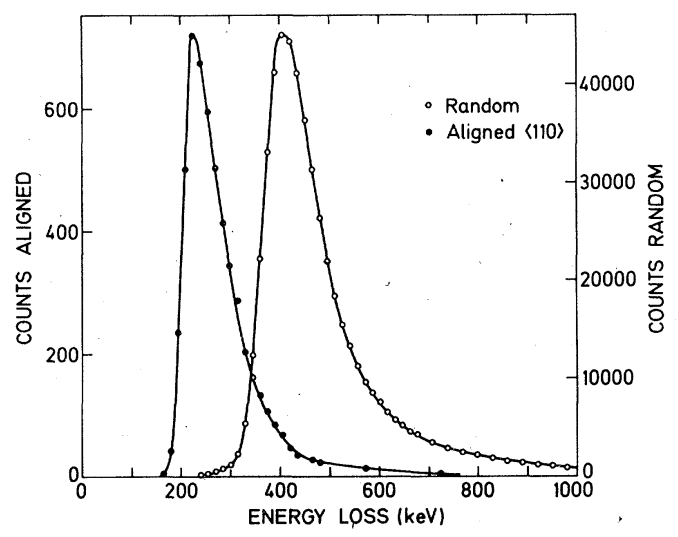

FIG. 6. Energy-loss spectra for $15-\mathrm{GeV} / c$ protons incident on a $0.74-\mathrm{mm}$ Ge single crystal. The dots correspond to particles channeled along a $\langle 110\rangle$ axis, and the circles correspond to particles incident in a "random" direction.

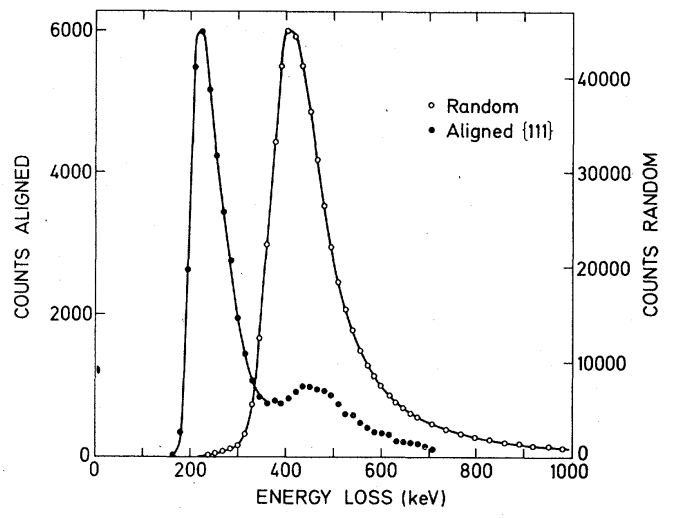

FIG. 7. Energy-loss spectra for $15-\mathrm{GeV} / c$ protons incident on the $0.74-\mathrm{mm}$ Ge single crystal. Similar to Fig. 6 except that the channeled spectrum corresponds to particles incident along a $\{111\}$ plane. The small high-energy peak on the channeled spectrum is perhaps due to channeling between a pair of close lying $\{111\}$ planes.

planes. In Figs. 10-13 are given similar results for a $900-\mu$ Si crystal.

All our experimental results of energy loss in a random direction for both positive and negative particles are summarized in Table IV, where, for each spectrum, the average energy loss $\langle\Delta E\rangle$, and the most probable energy loss $\Delta E_{p}$ are displayed. As expected, the results for positive and negative particles agree with each other. In Sec. III B 2 these results will be discussed and compared with the theoretical expectations.

The aligned spectra are characterized by being in general narrower than the random spectra, by having a very steep low-energy-loss side, andfor the planes-by having some structure in the

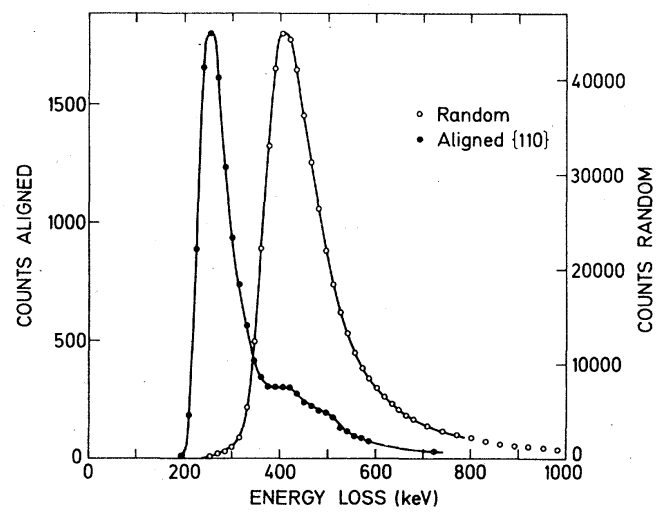

FIG. 8. Energy-loss spectra for $15-\mathrm{GeV} / c$ protons incident on the $0.74-\mathrm{mm}$ Ge single crystal. Similar to Fig. 6 except that the channeled spectrum corresponds to particles incident along a $\{110\}$ plane. 


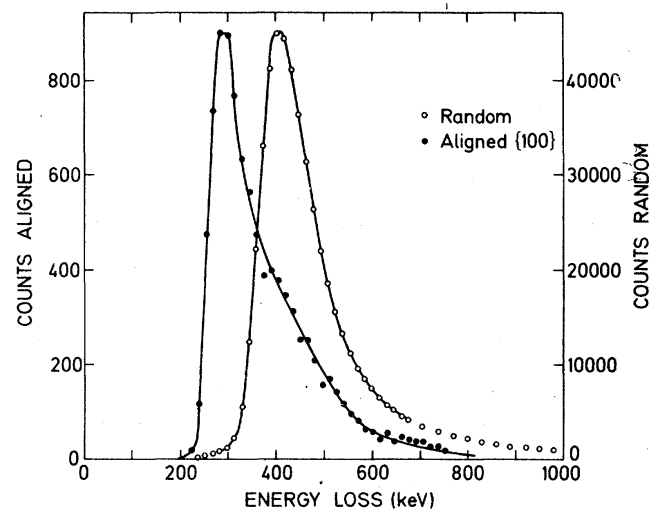

FIG. 9. Energy-loss spectra for $15-\mathrm{GeV} / c$ protons incident on the $0.74-\mathrm{mm}$ Ge single crystal. Similar to Fig. 6 except that the channeled spectrum corresponds to particles incident along the $\{100\}$ plane. This plane is rather weak, and the spectrum contains a relatively larger random component than the previous spectra.

tails. In most cases we ascribe the structure to a content of random-energy-loss particles.

In Fig. 6 we have plotted those particles which enter the crystal with an angle to the $\langle 110\rangle$ axis less than $\frac{1}{2} \psi_{1}$, where $\psi_{1}$ is Lindhard's critical angle $(=0.18 \mathrm{mrad}$ for $15-\mathrm{GeV} / c$ protons on $\mathrm{Ge}$; $\mathrm{cf}$. Table III), and leave the crystal with an angle less than $\frac{1}{4} \psi_{1}$ to the axis. We use the larger acceptance angle on the incident side, where the angular resolution is the poorer, in order to improve the statistics, whereas the exit criterion helps to select particles which have been well channeled through the crystal.

In Figs. 7-9 are plotted the energy-loss spectra

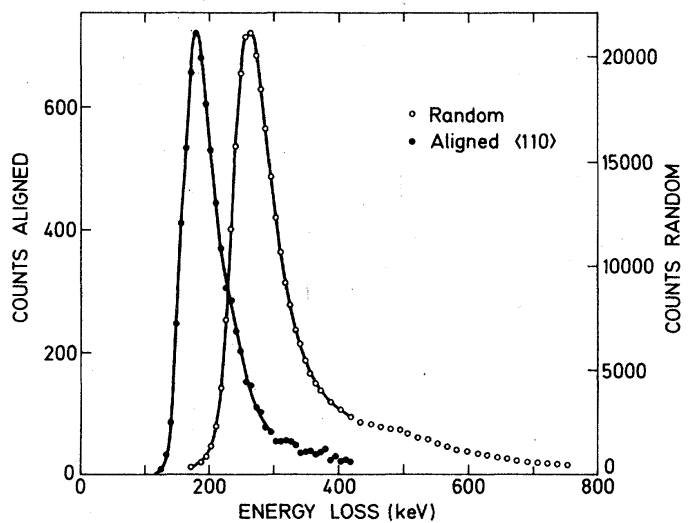

FIG. 10. Energy-loss spectra for $15-\mathrm{GeV} / c$ protons incident on a $0.90-\mathrm{mm} \mathrm{Si}$ single crystal. The dots correspond to particles channeled along a $\langle 110\rangle$ axis, and the circles to particles incident in a "random" direction.

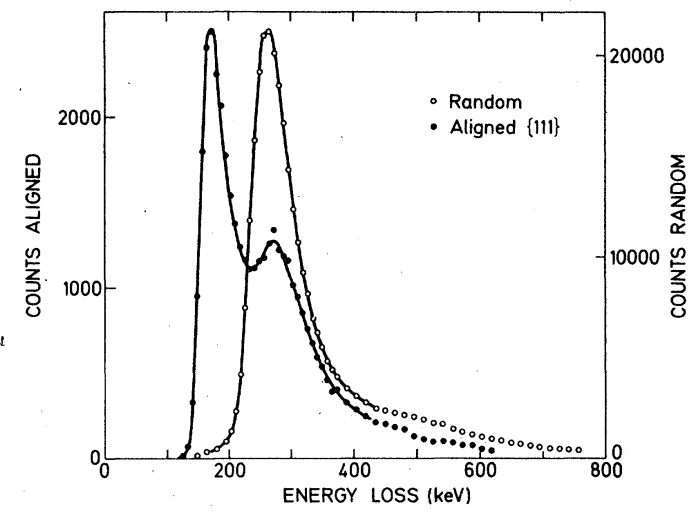

FIG. 11. Energy-loss spectra for $15-\mathrm{GeV} / c$ protons incident on a $0.90 \mathrm{~mm} \mathrm{Si}$ single crystal. Similar to Fig. 10 except that the channeled spectrum corresponds to particles incident along $\{111\}$ planes. As in Fig. 7 . there is a small high-energy peak probably due to channeling between close-lying $\{111\}$ planes.

for those particles which have incident and exit angles to the various planes of less than $0.05 \mathrm{mrad}$, which is of the order of magnitude of the critical angle for the planes. It is not possible to remove the "random" component in the spectra by further decreasing the incident and exit angles because of resolution problems (cf. Table II). Also crystal bending causes a certain fraction of random particles to be present in the spectra. For the $\{111\}$ planes we make a supplementary comment below.

The "best" experimental parameter with which to characterize the energy loss of well-channeled particles seems to be the position of the steep lowenergy-loss side of the spectrum. This parameter is proportional to crystal thickness and is virtually

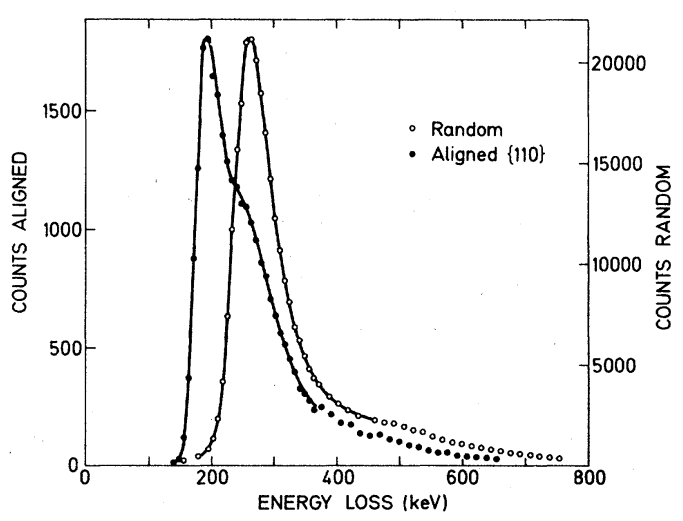

FIG. 12. Energy-loss spectra for $15-\mathrm{GeV} / c$ protons incident on a $0.90-\mathrm{mm}$ Si single crystal. Similar to Fig. 10 except that the channeled spectrum corresponds to particles incident along a $\{110\}$ plane. 


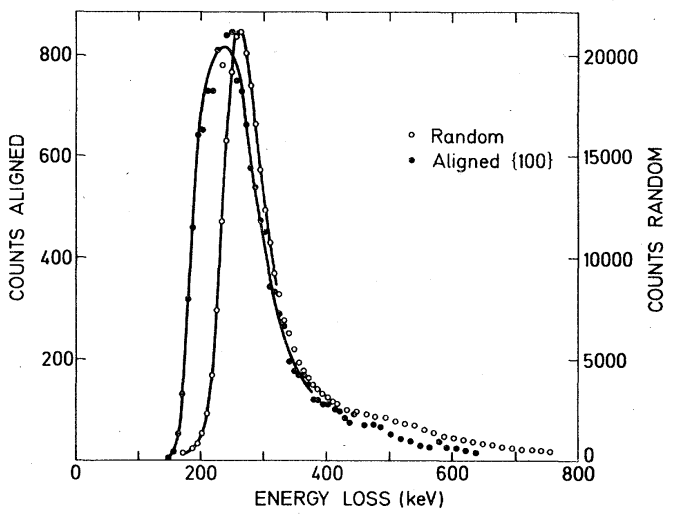

FIG. 13. Energy-loss spectra for $15-\mathrm{GeV} / c$ protons incident on a $0.90-\mathrm{mm}$ Si single crystal. Similar to Fig. 10 except that the channeled spectrum corresponds to particles incident along a $\{100\}$ plane. Here the relatively poor angular resolution combined with a rather large dechanneling results in a very broad "channeled" spectrum.

independent of incident- and exit-angle criteria. Also the peak position is nearly proportional to crystal thickness and rather insensitive to angle criteria as long as they are well below the critical angles. On the other hand, the width of the distribution and the tail are very sensitive to changes in incident and exit angles.

Accordingly, in Table $\mathrm{V}$, we summarize the experimental results for well-channeled particles by displaying the most probable energy loss $\Delta E_{p}$, and the low-energy loss corresponding to half-maximum. We call this quantity $\Delta E_{1 / 2}$.

For silicon, for which some results are shown in Figs. 10-13, the critical angles are smaller than for germanium by a factor $\sim \frac{2}{3}$. For this reason angular resolution problems become more serious, especially for the less dense planes (i.e., $\{100\})$ where the distribution is very broad because of a large component of random particles and because of dechanneling. For that particular case only $\Delta E_{1 / 2}$ is fairly well defined.

We do not, for our measurements, claim the very high accuracy which can be obtained for $\mathrm{MeV}$ protons. In Tables IV and V the uncertainties quoted contain contributions from counting statistics, energy calibration, variation in depletion thickness, etc. The thickness of the depletion layer was taken to be equal to the crystal thickness, which was uniform within $\pm 1 \%$. We expect the overall uncertainty in the numbers in Tables IV and $\mathrm{V}$ to be less than $\pm 5 \%$.

\section{Discussion of random energy loss}

The stopping power for relativistic particles is well known theoretically being given by the BetheBloch formula with various corrections. At high energies the most important correction is the socalled density effect, which gives a considerable reduction in stopping power at extreme relativistic energies. This effect is only present in condensed materials where the target electrons can act collectively. The density effect has to do with polarization of the electron gas of the substance. We refer the reader to Jackson, ${ }^{4}$ which discusses the density effect. Although measurements of the stopping power at very high energies are very important both for experimental and theoretical reasons, only few exist. A measurement in which the particle energy is determined accurately before and after traversing a foil is not easy to per-

TABLE IV. Measured most probable energy loss $\Delta E_{p}$ and average energy loss $\langle\Delta E\rangle$ in Ge and $\mathrm{Si}$, for random incidence. All numbers are $\mathrm{eV} / \mu$. Some numbers are missing due to bad statistics.

\begin{tabular}{|c|c|c|c|c|c|c|c|}
\hline & & \multicolumn{2}{|c|}{$2 \mathrm{GeV} / c$} & \multicolumn{2}{|c|}{$6 \mathrm{GeV} / c$} & \multicolumn{2}{|c|}{$15 \mathrm{GeV} / c$} \\
\hline & & $\Delta E_{p}$ & $\langle\Delta E\rangle$ & $\Delta E_{p}$ & $\langle\Delta E\rangle$ & $\Delta E_{p}$ & $\langle\Delta E\rangle$ \\
\hline \multirow{6}{*}{$\mathrm{Ge} 0.74 \mathrm{~mm}$} & $\pi^{+}$ & $580 \pm 20$ & & $590 \pm 20$ & $680 \pm 20$ & $580 \pm 20$ & $685 \pm 15$ \\
\hline & $\pi^{-}$ & & & $565 \pm 15$ & $670 \pm 15$ & $570 \pm 15$ & $680 \pm 15$ \\
\hline & $k^{+}$ & & & $570 \pm 30$ & $670 \pm 30$ & $550 \pm 20$ & $660 \pm 15$ \\
\hline & $k^{-}$ & & & $540 \pm 15$ & $645 \pm 15$ & $570 \pm 20$ & $680 \pm 15$ \\
\hline & $p$ & $590 \pm 20$ & & $560 \pm 20$ & & $555 \pm 20$ & $660 \pm 15$ \\
\hline & $\bar{p}$ & & & $525 \pm 15$ & $635 \pm 15$ & $550 \pm 25$ & $660 \pm 20$ \\
\hline \multirow{6}{*}{ Si $0.9 \mathrm{~mm}$} & $\pi^{+}$ & $295 \pm 5$ & $340 \pm 5$ & $295 \pm 5$ & $345 \pm 5$ & $295 \pm 5$ & \\
\hline & $\pi^{-}$ & $288 \pm 5$ & $340 \pm 5$ & $292 \pm 5$ & $346 \pm 5$ & $292 \pm 5$ & $353 \pm 5$ \\
\hline & $k^{+}$ & & & $282 \pm 5$ & $336 \pm 5$ & $292 \pm 8$ & \\
\hline & $k^{-}$ & & & $283 \pm 5$ & $338 \pm 5$ & $294 \pm 5$ & $352 \pm 5$ \\
\hline & $p$ & $315 \pm 5$ & $360 \pm 5$ & $280 \pm 5$ & $335 \pm 5$ & $290 \pm 5$ & \\
\hline & $\bar{p}$ & $292 \pm 10$ & $343 \pm 10$ & $279 \pm 5$ & $334 \pm 5$ & $285 \pm 5$ & $343 \pm 5$ \\
\hline
\end{tabular}


TABLE V. Measured most probable energy loss $\Delta E_{p}$ and low-energy loss $\Delta E_{1 / 2}$ for wellchanneled particles. All numbers are in $\mathrm{eV} / \mu$. Some numbers are missing due to bad statistics.

\begin{tabular}{|c|c|c|c|c|c|c|c|c|}
\hline & & & \multicolumn{2}{|c|}{$2 \mathrm{GeV} / c$} & \multicolumn{2}{|c|}{$6 \mathrm{GeV} / c$} & \multicolumn{2}{|c|}{$15 \dot{\mathrm{GeV}} / c$} \\
\hline & & & $\Delta E_{1 / 2}$ & $\Delta E_{p}$ & $\Delta E_{1 / 2}$ & $\Delta E_{p}$ & $\Delta E_{1 / 2}$ & $\Delta E_{p}$ \\
\hline \multirow{9}{*}{$\mathrm{Ge} 0.74 \mathrm{~mm}$} & \multirow{3}{*}{$\langle 110\rangle$} & $\pi^{+}$ & $270 \pm 10$ & $315 \pm 15$ & $285 \pm 10$ & $325 \pm 15$ & $300 \pm 10$ & $325 \pm 15$ \\
\hline & & $p$ & $230 \pm 10$ & $280 \pm 15$ & $245 \pm 10$ & $280 \pm 15$ & $270 \pm 10$ & $305 \pm 15$ \\
\hline & & $\pi^{-}$ & & & $625 \pm 20$ & $740 \pm 25$ & $635 \pm 20$ & $790 \pm 20$ \\
\hline & \multirow{2}{*}{111} & $\pi^{+}$ & $270 \pm 10$ & $285 \pm 15$ & $280 \pm 7$ & $315 \pm 15$ & $285 \pm 7$ & $325 \pm 15$ \\
\hline & & $p$ & $220 \pm 10$ & $240 \pm 15$ & $240 \pm 7$ & $260 \pm 15$ & $265 \pm 7$ & $296 \pm 15$ \\
\hline & \multirow{2}{*}{110} & $\pi^{+}$ & $295 \pm 15$ & $330 \pm 15$ & $320 \pm 10$ & $360 \pm 15$ & $325 \pm 10$ & $365 \pm 15$ \\
\hline & & $p$ & & & $280 \pm 10$ & $305 \pm 15$ & $305 \pm 7$ & $340 \pm 15$ \\
\hline & \multirow{2}{*}{100} & $\pi^{+}$ & & & $360 \pm 8$ & $395 \pm 15$ & $370 \pm 10$ & $405 \pm 20$ \\
\hline & & $p$ & & & $320 \pm 8$ & $345 \pm 15$ & $345 \pm 7$ & $395 \pm 15$ \\
\hline \multirow{9}{*}{ Si $0.9 \mathrm{~mm}$} & \multirow{3}{*}{$\langle 110\rangle$} & $\pi^{+}$ & $165 \pm 5$ & $190 \pm 5$ & $175 \pm 5$ & $193 \pm 5$ & $180 \pm 5$ & $193 \pm 5$ \\
\hline & & $p$ & $172 \pm 5$ & $190 \pm 5$ & $157 \pm 5$ & $182 \pm 5$ & $169 \pm 5$ & $198 \pm 5$ \\
\hline & & $\pi^{-}$ & $310 \pm 15$ & $355 \pm 20$ & $315 \pm 10$ & $385 \pm 15$ & $310 \pm 10$ & $400 \pm 15$ \\
\hline & \multirow{2}{*}{111} & $\pi^{+}$ & & & $170 \pm 5$ & $191 \pm 7$ & $171 \pm 7$ & $197 \pm 10$ \\
\hline & & $p$ & & & $157 \pm 5$ & $172 \pm 7$ & $166 \pm 5$ & $186 \pm 7$ \\
\hline & \multirow{2}{*}{110} & $\pi^{+}$ & & & $193 \pm 5$ & $216 \pm 10$ & $197 \pm 7$ & $218 \pm 10$ \\
\hline & & $p$ & & & $178 \pm 5$ & $200 \pm 8$ & $189 \pm 5$ & $211 \pm 10$ \\
\hline & \multirow{2}{*}{100} & $\pi^{+}$ & & & $213 \pm 10$ & $240 \pm 15$ & $220 \pm 10$ & \\
\hline & & $p$ & & & $203 \pm 5$ & $220 \pm 10$ & $210 \pm 10$ & \\
\hline
\end{tabular}

form. Another method is to measure the energy deposited in the foil by the particle. Nuclear emulsions have been widely used. The foil can also be a solid-state detector, which has the advantage of being inexpensive, small, and relatively easy to operate. It therefore lends itself easily to stopping-power measurements. At present, silicon, germanium, and some semiconducting alloys can be used for detector fabrication. To our knowledge, solid-state detectors have not been used to measure systematically the stopping powers of extremely relativistic particles before.

It is essential to realize that the energy loss of the particle in passing through the detector does not correspond exactly to the energy deposited in the sample, as measured by the total number of electron-hole pairs created. This is due to the fact that a particle may suffer hard collisions with electrons in the detector giving rise to energetic electrons ( $\delta$ rays) which may escape from the detector without depositing all their energy therein. This will especially influence the tail of the energyloss distribution. The measured spectrum of energy deposited in the crystal is therefore a "restricted" energy-loss spectrum in the sense that collisions in which energetic electrons are ejected, do not contribute fully.

Another interesting effect which will, in principle, influence the tail of the measured spectrum is nuclear reactions. At the present energies nuclear reactions may result in the production of several particles which (if charged) lose energy in passing through the detector. The energy deposited by such particles will show up in the detector as a high-energy pulse and therefore belong to the tail of the distribution. This effect is small and has furthermore been suppressed in the present experiments by the coincidence requirements. This demands that at least one particle hits the last scintillator SC4, corresponding to a maximum exit angle of $5 \mathrm{mrad}$, cf. Fig. 1.

At this stage we want to point out an interesting application of the effect: The number of particles giving rise to high-energy deposits in the detector will be correlated to the number of nuclear reactions taking place. It should therefore be possible to study nuclear-reaction cross sections by means of the very high-energy pulses in the solidstate detector. Results of such experiments--where the last scintillator SC4 was removed-will be published separately.

Fano $^{4}$ gives the following simple formula for the average restricted stopping power:

$$
\begin{aligned}
\left(-\frac{d E}{d x}\right)_{\text {rest }}=\frac{2 \pi e^{4} N Z_{2}}{m v^{2}}[ & 2 \ln \frac{2 m v^{2} \gamma^{2}}{I}-2 \beta^{2}-\delta \\
& -\left(\frac{T_{0}}{2 m c^{2} \gamma^{2}}-\beta^{2}\right. \\
& \left.\left.-\ln \frac{T_{0}}{2 m v^{2} \gamma^{2}}\right)\right],
\end{aligned}
$$


where the term in parentheses represents a modification of the normal energy loss due to a lack of contributions from energy transfers greater than $T_{0}$. In the formula, $\beta$ and $\gamma$ are the usual relativistic parameters $v / c$ and $1 /\left(1-v^{2} / c^{2}\right)^{1 / 2}$, respectively, $I$ is the mean-excitation potential of the stopping substance, and $\delta$ is the density-effect correction. Corrections due to shell effects and Čerenkov-radiation loss, are very small in the present case, and are neglected.

In order to evaluate $T_{0}$, one should first determine the kinematics of particle-electron collisions, secondly, electron energy-range tables should be consulted to determine, as a function of crystal depth, those electrons that are likely to escape from the crystal, and, thirdly, an average over depths in the crystal should be performed. This leads to an estimate of $T_{0}$ of the order of $500 \mathrm{keV}$ for the $740-\mu \mathrm{Ge}$ and $900-\mu \mathrm{Si}$ crystal. We expect the uncertainty in $T_{0}$ determined this way to be $\sim 30 \%$, but this uncertainty results only in $a \sim 1 \%$ uncertainty in the stopping power of Eq. (2). The $I$ values we have used are those suggested by Andersen and Ziegler, ${ }^{8}$ namely, $I_{\mathrm{Si}}=165 \mathrm{eV}$ and $I_{\mathrm{Ge}}$ $=330 \mathrm{eV}$. As regards the density corrections, we use the theoretical results of Sternheimer. ${ }^{5}$

The theoretical energy loss spectrum is given by Landau's theory ${ }^{9}$ with Bethe's value for the average energy loss from Eq. (2) (without restriction). The Landau distribution is very simple, depending on only one parameter $\lambda$, i.e.,

$$
\lambda=\frac{\Delta-\bar{\Delta}}{\xi}-\beta^{2}-0.423-\ln \kappa
$$

where $\Delta$ is the energy loss, $\bar{\Delta}$ is the average energy loss, and

$$
\begin{aligned}
& \xi=\left(2 \pi e^{4} / m v^{2}\right) N Z_{2} t, \\
& \kappa=\xi / \boldsymbol{T}_{\max } .
\end{aligned}
$$

$t$ is the thickness of the sample, $T_{\max }$ is the maximum energy transfer to an electron $\left(T_{\max } \approx 2 m v^{2} \gamma^{2}\right)$. For Landau's theory to be valid, the parameter $\kappa$ should not exceed 0.01 . This criterion is fulfilled for the present case. The maximum for the Landau curve is found for $\lambda=-0.2$.

The measured spectra are expected to differ from the Landau spectrum especially in the tail where the measured spectrum should be the lower due to the escape of the $\delta$ rays produced by the high-energy-loss particles. In Fig. 14 we compare a measured spectrum to a Landau spectrum designed to have the theoretical average energy loss (without restriction). The agreement seems fair, although the measured spectrum is somewhat broader than the theoretical one. Detector resolution or nonuniformity in the depletion thickness

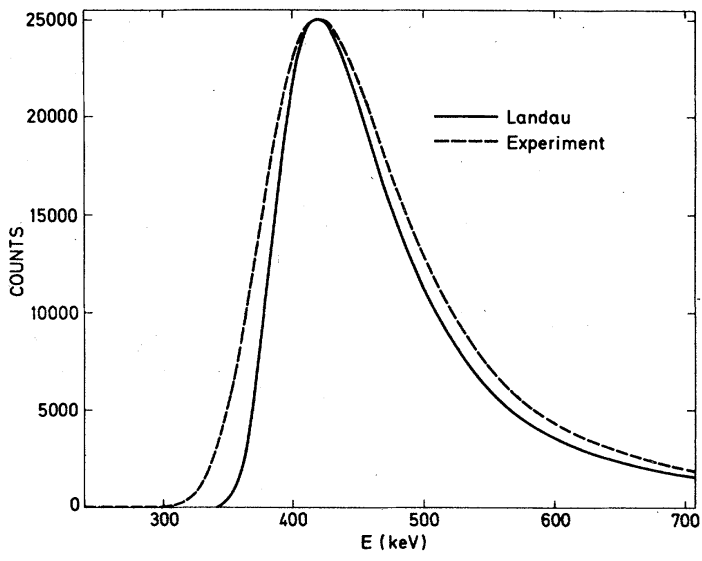

FIG. 14. Comparison between a measured energydeposit spectrum for $6-\mathrm{GeV} / c \pi^{-}$incident on the 0.74- $\mathrm{mm}$ Ge single crystal and a theoretical Landau energy-loss spectrum normalized to the same height.

are too small to account for this. We believe that energetic electrons ( $E \gtrsim 50 \mathrm{keV}$ ) formed close to the back of the crystal contribute considerably to the broadening of the experimental spectra. Such electrons may or may not escape and will thus deposit varying amounts of energy in the detector. In fact, the relative broadening of the experimental spectra compared to the Landau spectrum is large for very thin crystals $(280 \mu)$, and very small for a $4.2-\mathrm{mm}$ crystal.

In Figs. 15 and 16 we summarize our results

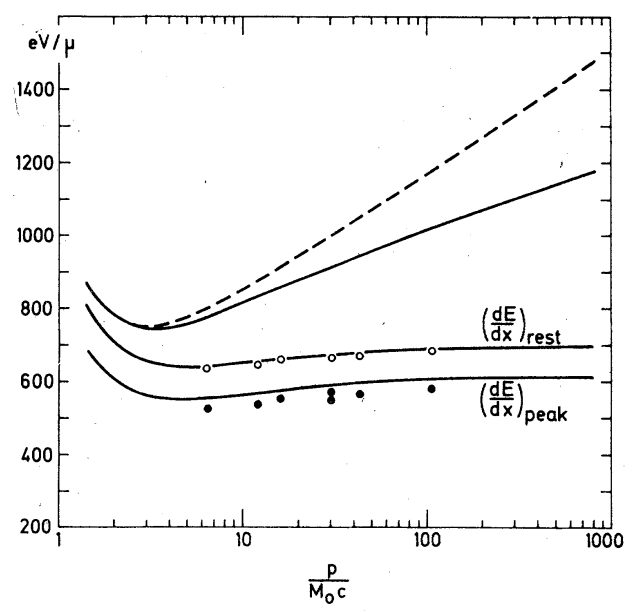

FIG. 15. Random energy loss in germanium. The curves are, from top: average stopping power without (dashed curve) and with (full curve) density effect correction. Average stopping power with both restriction and density effect taken into account; open circles are experimental points. Theoretical position of the peak of the energy-loss spectrum compared to experimental points (filled circles). 


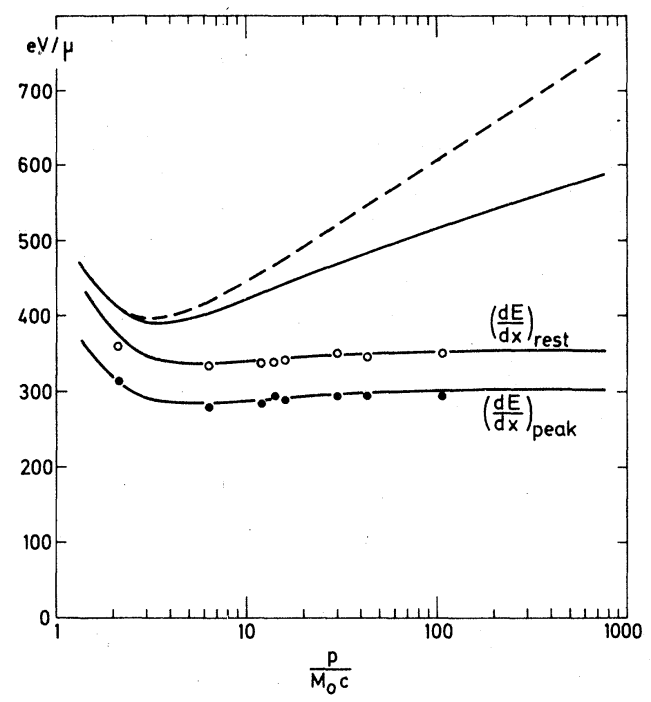

FIG. 16. Random energy loss in silicon. Completely analogous to Fig. 15.

for random particles. We show theoretical curves for the average energy loss, unrestricted, with and without density-effect correction--to give an impression of the size of the density effect. We also show the restricted energy loss compared to measurements, and finally the theoretical peak of the distribution compared to measurements. We plot the quantities versus a parameter $p / M_{0} c=\beta \gamma$ which is convenient both theoretically and experimentally.

\section{Energy loss for aligned positive particles}

Several authors ${ }^{7 \cdot 10^{-16}}$ have attempted to describe the energy loss of channeled particles theoretically. The early theories were based on the argument that, asymptotically, there is equipartition between contributions to stopping from close and distant collisions. The stopping of aligned particles in this picture has two sources: a standard contribution from the valence electrons, and a contribution from core electrons where close collisions are more or less suppressed. An important task in the theories is to evaluate the core electron contribution as accurately as possible. None of the theories are readily applicable to extreme relativistic particles. Lindhard originally proposed a semiquantitative formula for the stopping as function of particle position $\vec{b}$ in the transverse space (transverse with respect to the axis or planar direction in question)

$$
\frac{d E}{d x}(\overrightarrow{\mathrm{b}})=\left(\frac{d E}{d x}\right)_{\text {random }}\left((1-\alpha)+\alpha \frac{Z(\overrightarrow{\mathrm{b}})}{Z_{2}}\right),
$$

where $\alpha$ is a parameter between $\frac{1}{2}$ and 1 , tending to $\frac{1}{2}$ for very high velocities, and $N Z(\vec{b})$ is the local electron density at position $\vec{b}$, averaged along a straight-line trajectory parallel to the axis or plane.

From Lindhard's formula one finds for not too high velocities with $\alpha>\frac{1}{2}$ a stopping which is smaller than half the random value. For very high velocities - as in the present case $-\alpha$ should equal $\frac{1}{2}$ and the formula reads

$$
\frac{d E}{d x}(\overrightarrow{\mathrm{b}})=\left(\frac{d E}{d x}\right)_{\text {random }} \frac{Z_{2}+Z(\overrightarrow{\mathrm{b}})}{2 Z_{2}} .
$$

In this region the Lindhard expression therefore predicts a stopping somewhat higher than $50 \%$ of random. Although this formula was not expected to be strictly valid, it gives qualitatively the same results as the experiments: The denser channels (whether axial or planar) have the larger stopping. Experiments ${ }^{10}$ showed that $\alpha$ could not be assigned the asymptotic value $\frac{1}{2}$ for protons of energies as high as $\sim 10 \mathrm{MeV}$. Theoretical work was then concentrated upon the problem of explaining the channeled stopping power for protons at a few $\mathrm{MeV}$.

In the most recent paper by Dettman ${ }^{16}$ the average energy loss in a single atomic collision as a function of impact parameter is calculated. Afterwards, contributions from the atoms in the lattice are added together. For the valence electrons a free-electron-gas model is applied, whereas core electrons are treated using Hartree-Fock wave functions. In the limit of high velocities, however, Dettmann predicts a channeled stopping power identical to the one given by Eq. (6)

Recently, Esbensen and Golovchenko $0^{6}$ have treated the stopping power for channeled particles. These authors also find the average energy loss to an atom, as function of impact parameter, using standard first-order perturbation theory. They need not distinguish between core and valence electrons and they utilize the reciprocal lattice in order to add contributions from atoms in the crystal.

In the following we shall primarily compare our results with the theory of Esbensen and Golovchenko and thereby elucidate the theoretical controversy about the energy loss of channeled particles in the limit of high velocities. For high velocities $\left(\gamma v>Z_{2} e^{2} / \hbar\right)$, Esbensen and Golovchenko derive the following formula for the path-dependent stoppingpower due to electronic excitations (nonrelativistic)

$$
\frac{d E}{d x}(\overrightarrow{\mathrm{b}})=\frac{2 \pi Z_{1}^{2} e^{4}}{m v^{2}} N\left[\left[Z_{2}+Z(\overrightarrow{\mathrm{b}})\right] \ln \left(\frac{2 m v^{2}}{I}\right)+C(\overrightarrow{\mathrm{b}})\right],
$$

where

$$
C(\overrightarrow{\mathrm{b}})=\sum_{\vec{G} \neq \vec{D}} e^{i \overrightarrow{\mathrm{G}} \cdot \overrightarrow{\mathrm{b}}} \rho(G) \ln \left(\frac{2 m I}{\hbar^{2} G^{2}}\right),
$$


and

$$
\rho(G)=\left\langle 0\left|\sum_{j=1}^{z_{2}} e^{-i \vec{G} \cdot \vec{r}_{j}}\right| 0\right\rangle
$$

is the Fourier transform of the electronic charge density in the target atoms. $\vec{b}$ is the position of the penetrating particle in transverse space (transverse with respect to the axial or planar direction in question), and the sum over $\vec{G} \neq \overrightarrow{0}$ includes all nonzero, reciprocal-lattice vectors in the transverse space. We note that the contribution from $C(\vec{b})$ vanishes if we average over all positions $\vec{b}$ in transverse space, while $Z(\vec{b})$ will be replaced by $Z_{2}$, i.e., Bethe's formula is contained in $\mathrm{Eq}$. (7).

Note also that the term $C(\vec{b})$ is velocity independent, which means that Eq. (7) becomes identical to Eq. (6) in the limit where $C(\vec{b})$ can be neglected. As we shall see below, this limit is never reached and the correction $C(\vec{b})$ is substantial even for $15-\mathrm{GeV} / c$ pions. But first let us include some important relativistic corrections.

From the study of random stopping it is well known that the contribution from transverse excitations (resulting from the vector potential part of the electron-particle interaction) satisfies a strict equipartition with equal contributions from very low - and very high-energy transfers. ${ }^{4}$ We can include the contribution from very high-energy transverse excitations in Eq. (7) as a path-dependent correction, while the contribution from very low-energy transfers results in a random pathindependent correction. This is achieved in Eq. (7) by replacing $\ln \left(2 m v^{2} / I\right)$ by $\left\{\ln \left[2 m(\gamma v)^{2} / I\right]\right.$ $\left.-\beta^{2}\right\}$. We finally include the density effect, which is important in condensed matter, as a random path-independent correction, since it reduces the contribution from the low-energy transverse excitations. ${ }^{4}$ Thus we obtain in the relativistic regime

$$
\begin{aligned}
\frac{d E}{d x}(\overrightarrow{\mathrm{b}})=\frac{2 \pi Z_{1}^{2} e^{4}}{m v^{2}} N\{ & {\left[Z_{2}+Z(\overrightarrow{\mathrm{b}})\right] } \\
& \times\left[\ln \left(\frac{2 m(\gamma v)^{2}}{I}\right)-\beta^{2}\right] \\
& \left.-Z_{2} \delta+C(\overrightarrow{\mathrm{b}})\right\} .
\end{aligned}
$$

We have used the form factors (i.e., the Fourier components of the electronic charge density) given by Doyle and Turner ${ }^{17}$ to calculate $Z(\vec{b})$ and $C(\vec{b})$ for the various axial and planar directions. These form factors have been determined for free atoms using relativistic Hartree-Fock atomic wave functions. Form factors for the solid state would of course be more appropriate, and we therefore

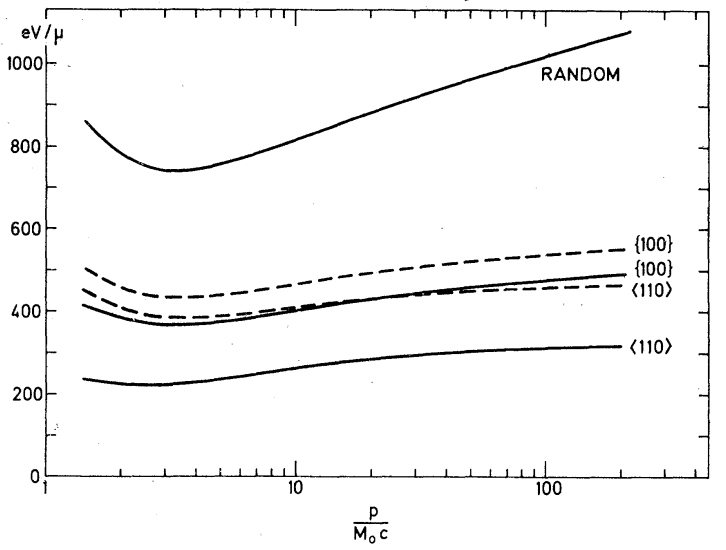

FIG. 17. Theoretical curves for the average energy loss in germanium showing influence of the correction $C(\vec{b})$. The correction is strong for the $\langle 110\rangle$ axis, and less important for a weak plane like the $\{110\}$ plane. Full-drawn curves include the correction, whereas dashed curves do not.

present our calculations with some reservation.

In Fig. 17 we compare the calculated mean energy loss of best channeled particles in a $\langle 110\rangle$ axial and $a\{100\}$ planar direction in Ge, with (fulldrawn curves) and without (dashed curves) the correction $C(\vec{b})$ in $\mathrm{Eq}$. (10). The random stopping power is also shown. The correction $C(\vec{b})$ is seen to be essential and we shall therefore always include it in the following.

In connection with the experimental results, we noted that the low-energy side of the recorded aligned spectra are very steep and insensitive to the choice of incident- and exit-angle criteria, whereas the tails of the spectra are sensitive to angle criteria. The tail of an aligned spectrum is therefore due to particles with quite different trajectories inside the crystal. The energy loss of best channeled particles can only be extracted from the low-energy side of the spectrum and as a representative value we use the energy loss at half-maximum $\Delta E_{1 / 2}$.

For the very high energies (and thin crystals) with which we are concerned, dechanneling is nearly negligible, cf. Ref. 3, Fig. 14, where the influence of dechanneling on wide-angle scattering yield is calculated for $1.15-\mathrm{GeV} / c$ protons, based on a diffusion equation. ${ }^{18}$ For the present energies the dechanneling will be even less important. We therefore expect the increase in transverse energy of very well-channeled particles to be negligibly small in traversing the crystal. A direct comparison between our measurements and the theoretical calculations for the best channeled particles should therefore be justified.

Let us recall that the Landau distribution in the 
random case is constructed to give the right mean value [cf. Eq. (3)], whereas the shape of the distribution is determined by close collisions. In the same manner we can construct an energy-loss distribution for the best channeled particles from the local electron density $Z(\vec{b}) N$ (which is averaged along the particle trajectory) and with the mean value given by Eq. (10). In particular, the energy loss (per path length) at half-maximum on the lowenergy side of a local Landau distribution (this corresponds to choosing $\lambda=-1.6$ ) is

$$
\begin{aligned}
\left(\frac{d E}{d x}(\overrightarrow{\mathrm{b}})\right)_{1 / 2}= & \frac{d E}{d x}(\overrightarrow{\mathrm{b}})+\frac{2 \pi Z_{1}^{2} e^{4}}{m v^{2}} Z(\overrightarrow{\mathrm{b}}) \\
& \times N\left\{-1.18+\beta^{2}+\ln [\kappa(\overrightarrow{\mathrm{b}})]\right\},
\end{aligned}
$$

with

$$
\kappa(\overrightarrow{\mathrm{b}})=\left(2 \pi Z_{1}^{2} e^{4} / m v^{2}\right)\left[Z(\overrightarrow{\mathrm{b}}) N t / T_{\max }\right],
$$

cf. Eqs. (3) and (4).

In Figs. 18-20 we compare this result for the best channeled particles (full-drawn curves) for the $\langle 110\rangle$ axial and the $\{111\},\{110\}$, and $\{100\}$ planar directions in a $740-\mu$ Ge and a $900-\mu$ Si crystal with the experimental results $\Delta E_{1 / 2} t^{-1}$, defined previously. The results in a random direction are also shown.

We do not claim that these theoretical and experimental quantities should correspond exactly to one another, but keeping in mind that the experimental value $\Delta E_{1 / 2}$ essentially covers all values on the low-energy-loss side of the aligned spectrum within $10 \%$, the agreement between theory

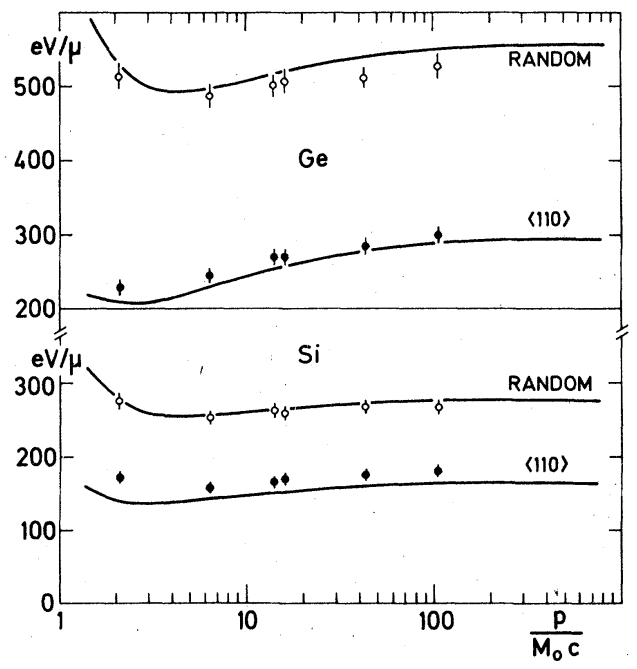

FIG. 18. Comparison between theory and experiment for leading edge $\left(\Delta E_{1 / 2}\right)$ in random direction and in the $\langle 110\rangle$ axial direction for both $0.74-\mathrm{mm}$ Ge and $0.9-\mathrm{mm}$ Si crystals.

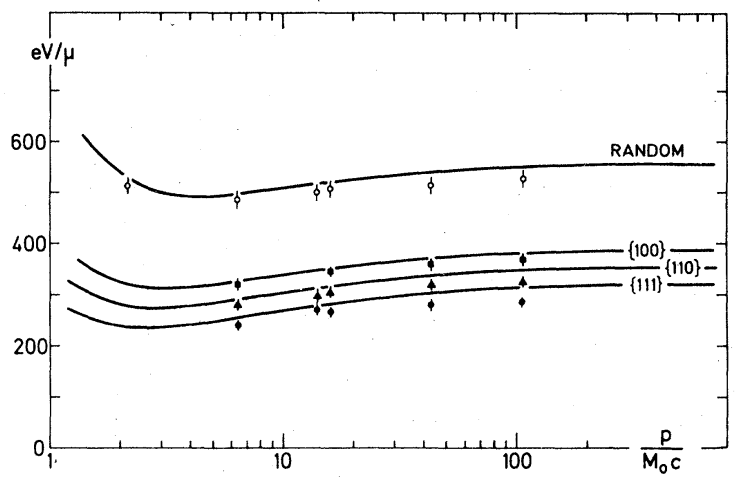

FIG. 19. Comparison between theory and experiment for leading edge $\left(\Delta E_{1 / 2}\right)$ for three planes in the $0.74-\mathrm{mm}$ Ge crystal. Random is also shown.

and experiment is satisfactory. The error bars in Figs. 18-20 do not include this uncertainty. It should be emphasized that the correction $C(\vec{b})$ is essential to obtain agreement between theory and experiment, cf. Fig. 17. The curves in Figs. 1820 tend towards a constant for increasing particle momentum as a consequence of both the density effect and the fact that only quantities like $\Delta E_{1 / 2}$ are suitable to characterize the energy loss of the best channeled particles. Therefore the simple limiting result of Eq. (6) based on equipartition rules only is, partly for experimental reasons, not very relevant.

It is interesting to note the difference of $\sim 10 \%$ in best channeled energy loss for $15-\mathrm{GeV} / c$ protons and pions along the $\langle 110\rangle$ axis in Ge. Had this difference been larger, i.e., comparable to the width of the spectra, one might consider using the effect for particle identification. In a previous paper ${ }^{3}$ a very narrow spectrum was observed for $1.35-\mathrm{GeV} /$ $c$ protons along $\{111\}$ planes in Ge.

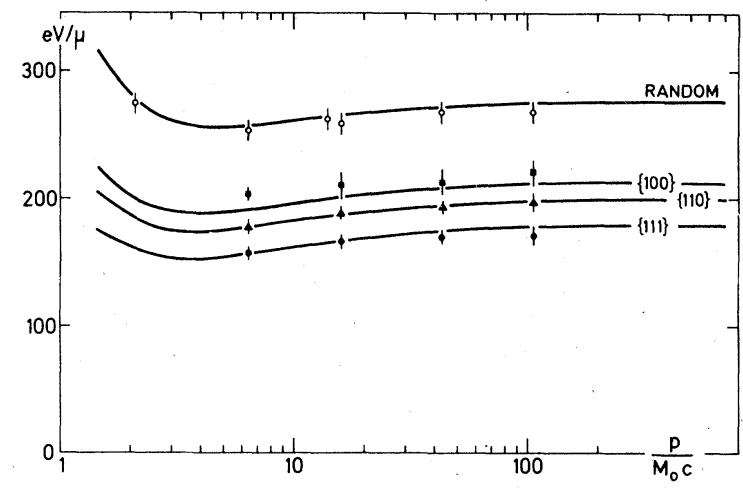

FIG. 20. Comparison between theory and experiment for leading edge $\left(\Delta E_{1 / 2}\right)$ for three planes in the 0.90 $\mathrm{mm} \mathrm{Si} \mathrm{crystal.} \mathrm{Random} \mathrm{is} \mathrm{also} \mathrm{shown.}$ 


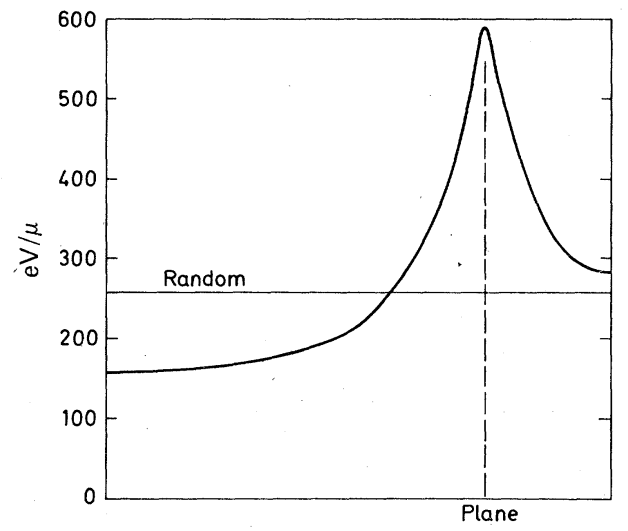

FIG. 21. Theoretical $\Delta E_{1 / 2}$ as function of distance to the $\{111\}$ planes in Si. The left-hand side of the figure corresponds to particles in the middle of the wide planar channel, whereas the right-hand side corresponds to particles in the middle of the narrow channel. Such particles should therefore experience an energy loss slightly above random.

Note from a comparison of Fig. 17 with Figs. 18-20 that the average energy loss (plotted in Fig. 17) does not differ much from $\Delta E_{1 / 2}$ in the case of the strong $\langle 110\rangle$ axis, i.e., the "local" Landau distribution is very narrow, as was to be expected. On the other hand, for the rather weak plane $\{100\}$ there is a considerable difference between the average energy loss and $\Delta E_{1 / 2}$ corresponding to the fact that the Landau distribution in this case is much broader than in the former.

In Figs. 7 and 11 there was a peak in the spectrum for particles aligned to the $\{111\}$ planes corresponding to an energy deposit slightly above the random peak. Actually the $\{111\}$ planes have a double structure: There is a set of widely spaced atomic planes (2.35- $\AA$ separation) bordered by a narrow set (0.78- $\AA$ separation).

In Fig. 21 the theoretical value of $\Delta E_{1 / 2} / t$ as function of position perpendicular to the $\{111\}$ planes in Si is shown. Theoretically, it is to be expected that particles channeled between the close-lying $\{111\}$ planes show a larger than random energy loss.

Ideally the ratio of the number of particles in the two planar channels should be $3: 1$, but for several reasons we do not expect to see this in the experimental spectra. First, the energy-loss spectra will be different, especially in the tails; second, dechanneling will be most serious for the narrow planes; and third, angular-resolution problems will smear the effects. At the present stage we can only conclude that our measurements indicate a content of particles channeled between the close $\{111\}$ planes. This has also been found, with good statistics, for $\mathrm{He}$ ions at $\mathrm{MeV}$ energies by Holland and Appleton. ${ }^{19}$

\section{Energy loss for aligned negative particles}

For negative particles it has been expected that one would see an increased energy loss for channeled particles compared to random since the channeled negative particles are attracted by the negative string potential and may be trapped in the potential. The particles then move in a region of high electron density and consequently that part of the energy loss which is associated with close collisions with electrons may be expected increased over the random value.

On the other hand, because of the strong dechanneling for particles close to the strings, negative particles can hardly remain close to strings for a longer path. Therefore, to be able to see an increased energy loss for negative particles, one should look at very thin crystals, and use small incidence angles to the axis. An anomalous energy-loss spectrum for electrons in a thin $\mathrm{Ge}$ crystal has been observed by Tomimasu et al. ${ }^{20}$

In Fig. 22 we show energy-loss spectra for 15$\mathrm{GeV} / c \pi^{-}$incident on a $2-\mathrm{mm}$ Ge crystal. Three spectra are shown corresponding to different angles of incidence to the $\langle 110\rangle$ axis. The spectrum for incidence angles larger than $1.2 \mathrm{mrad}$ is a random spectrum. There is a small shift towards higherenergy loss for particles incident at angles between two and three times the critical angle and a distinct shift of the whole spectrum for particles aligned with the axis.

In Fig. 23 we have shown the spectrum for well aligned $\pi^{-}$in the $0.74-\mathrm{mm}$ Ge crystal. Incident and exit angles are less than $\frac{1}{2} \psi_{1}$ and $\frac{1}{4} \psi_{1}$, respec-

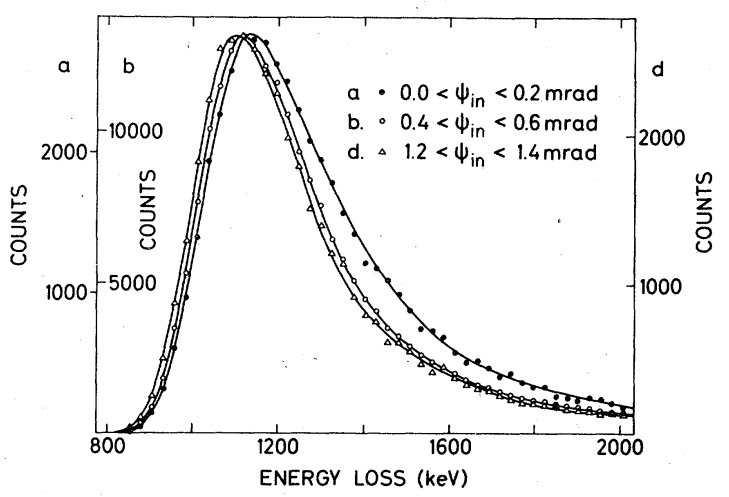

FIG. 22. Energy-loss spectra for $15-\mathrm{GeV} / c \pi^{-}$ traversing a $2.0-\mathrm{mm}$ Ge crystal. Particles incident with a small angle to the $\langle 110\rangle$ axis (a) give a spectrum which is shifted to higher energy loss. The effect is most marked in the tail. 


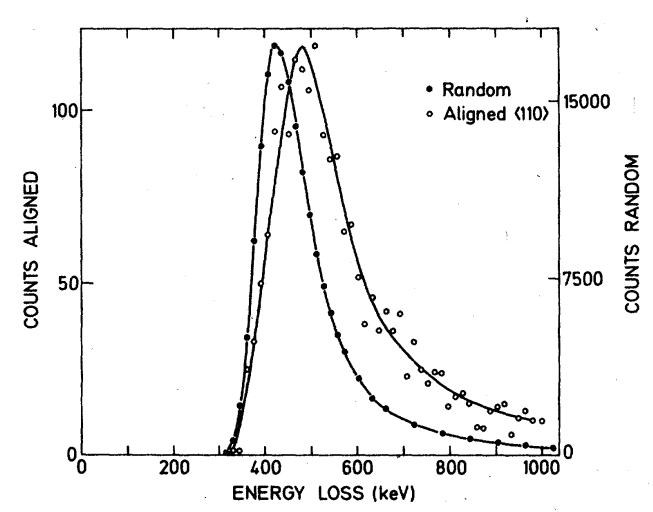

FIG. 23. Energy-loss spectra for $15-\mathrm{GeV} / c \pi^{-}$traversing the $0.74-\mathrm{mm}$ Ge crystal. For the aligned particles the angle to the $\langle 110\rangle$ axis is less than $\frac{1}{2} \psi_{1}$ on the incident side and less than $\frac{1}{4} \psi_{1}$ on the exit side of the crystal.

tively. For comparison the random spectrum is also shown. There is very marked increase in stopping as compared to random: the peak is shifted by $\sim 10 \%$ and the tail is much higher and longer. The average value is larger than random by more than $20 \%$. It is also apparent that dechanneling is strong since very few particles contribute to the spectrum (of the order of a few per mille of the events recorded).

Figure 24 shows the same for $15-\mathrm{GeV} / c \pi^{-}$incident on $0.9-\mathrm{mm}\langle 110\rangle \mathrm{Si}$. Again there are more random particles in the spectrum than for Ge but the effect is still undoubtedly there. We have not seen any effects of planes for negative particles. The energy-loss spectrum of particles incident along planar directions is identical to a randomenergy-loss spectrum.

Some of our preliminary results for negative particles have been published in a recent letter. ${ }^{21}$ Increased energy loss for $\pi^{-}$has also recently been

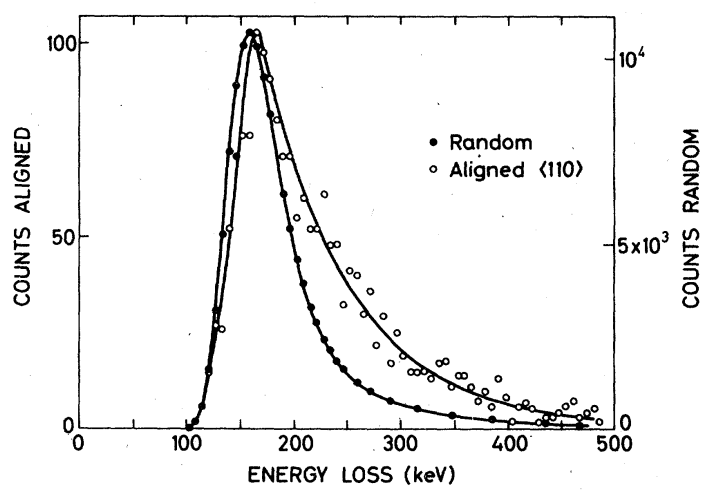

FIG. 24. Energy-loss spectra for $15-\mathrm{GeV} / c \pi^{-}$traversing the $0.90-\mathrm{mm} \mathrm{Si}$ crystal. Analogous to Fig. 23. found by Gemmell et al. ${ }^{22}$ for energies around 200 $\mathrm{MeV}$. Thus while this clear evidence of increased energy loss for negative particles, which in turn implies a focusing near atomic strings may be noted, we shall not try to present a detailed theoretical discussion of the results. The reason is that there is not-as for positive particles_any characteristic trajectory with which to associate any aspect of energy-loss distribution. Any detailed energy-loss calculation would involve an average over many different types of trajectories. The statistics (i.e., fluctuations) in the distribution will then depend on both the distribution of trajectories and the distribution of electron excitations along each trajectory. The effect of the latter on the width of the final distribution is not expected to be small because the hard collision responsible for the increased energy loss are also the ones occurring with the greatest fluctuations in number due to their relative infrequency. Nor are the contributing trajectories likely to be of a simple nature due to the intense dechanneling influence associated with close approach to the strings.

Preliminary computer simulations of $\pi^{-}$channeling in germanium show that, for small angles of incidence to the axis, small impact parameters are strongly favored compared to the case of random incidence. For angles of incidence somewhat larger than $\psi_{1}$ this effect disappears. It is apparent that channeled negative pions have a tendency to move close to the axis thereby suffering a larger than random energy loss, in accordance with the experimental observations.

\section{CONCLUDING REMARKS}

Although the critical angles for channeling are very small at these high energies, the intrinsic $\mathrm{Ge}$ and $\mathrm{Si}$ solid-state detectors are so perfect that it has proved rather easy by the present technique, and without the use of the computer, to align the crystal to the beam and establish the channeling conditions. The crucial point was the use of the crystal as an energy-loss detector. These detectors are well suited for the measurement of energy-loss spectra and our results for the random energy loss are in good accordance with the BetheBloch-Landau description.

For positive particles the influence of channeling on the energy loss is very pronounced. We have seen a strong reduction in stopping for channeled particles, and the energy-loss distributions are narrower than random-energy-loss distributions by a factor of $\sim 2$. A distinct difference in stopping along various crystallographic planes and axes is observed in contradiction to Dettmann's predic- 
tions for high-energy particles. Our experimental results are in good agreement with calculations of Esbensen and Golovchenko.

Finally, for negative particles, channeling results in an increased stopping. The observed energy-loss distributions are wider than the corresponding random energy-loss distributions and show $20 \%-25 \%$ increase in average energy loss.

\section{ACKNOWLEDGMENTS}

The authors are grateful to J. U. Andersen, J. Lindhard, and K. O. Nielsen for fruitful discussions and moral support, and to A。G. Wagh for help during one of the runs. The meticulous work of R. Bouclier, G. Million, and J. C. Santiard, who constructed the drift chambers and the attached electronics, and of R. Stuck, who manufactured the crystal cooling system, is gratefully acknowledged. The computer program simulating $\pi^{-}$channeling was kindly placed at our disposal by U. Schiebel. We are indebted to him, E. Worm, and S. Kjar Andersen for help in running the program. The Danish participation in the collaboration was made possible by funds from the Danish Committee for Accelerator Physics.
*Permanent address: Bell Laboratories, Murray Hill, N. J. 07974.

${ }^{1}$ O. Fich, J. A. Golovchenko, K. O. Nielsen, E. Uggerh $\phi j$, G. Charpak, and F. Sauli, Phys. Lett. B 57, 90 (1975).

${ }^{2}$ O. Fich, J. A. Golovchenko, K. O. Nielsen, E. Uggerh $\phi j$, C. Vraast-Thomsen, G. Charpak, S. Majewski, and F. Sauli, Phys. Rev. Lett. 36, 1245 (1976).

${ }^{3}$ H. Esbensen, O. Fich, J. A. Golovchenko, K. O. Nielsen, E. Uggerh $\phi j$, C. Vraast-Thomsen, G. Charpak, S. Majewski, F. Sauli, and J. P. Ponpon, Nucl. Phys. B 127, 281 (1977).

${ }^{4}$ U. F ano, Ann. Rev. Nucl. Sci. 13, 67 (1963). J. D. Jackson, Classical Electrodynamics (Wiley, New York, 1962), Chap. 13.

${ }^{5}$ R. M. Sternheimer, Phys. Rev. 145, 247 (1966).

${ }^{6} \mathrm{H}$. Esbensen and J. A. Golovchenko, Nucl. Phys. A 298, 382 (1978); H. Esbensen, Ph.D. thesis (University of Aarhus, 1977).

${ }^{7} J$. Lindhard, Mat. Fys. Medd. Dan. Vid. Selsk. 34, 14 (1965).

${ }^{8}$ H. H. Andersen and J. F. Ziegler, Hydrogen Stopping Powers and Ranges in all Elements (Pergamon, New York, 1977).

${ }^{9}$ L. D. Landau, J. Phys. USSR 8 , 201 (1944).

${ }^{10}$ B. R. Appleton, C. Erginsoy, and W. M. Gibson, Phys. Rev. 161, 330 (1967); C. Erginsoy, in Solid State Physics Research with Accelerators, Brookhaven National Laboratory Report No. BNL-50083 p. 30 (1968), (unpublished).

${ }^{11}$ M. Luntz and R. H. Bartram, Phys. Rev. 175, 468 (1968). M. Luntz and G. M. Heymsfield, Phys. Rev.
B 6, 2530 (1972).

${ }^{12}$ M. A. Kumakhov, Sov. Phys. Solid State 10, 2854 (1968). F. F. Komarov and M. A. Kumakhov, Radiat. Eff. 22, 1 (1974).

${ }^{13} \mathrm{~F}$. Bonsignori and A. Desalvo, Nuovo Cimento Lett. 1 , 589 (1969). F. Bonsignori and A. Desalvo, J. Phys. Chem. Solids 31, 2191 (1970).

${ }^{14}$ Y. H. Ohtsuki, Phys. Lett. A 34, 104 (1971); Y. H. Ohtsuki, M. Mizymo, and M. Kitagawa, J. Phys. Soc. Jpn. 31, 1109 (1971). M. Kitagawa and Y. H. Ohtsuki, Phys. Rev. B $\underline{5}, 3418$ (1972).

${ }^{15}$ Yu. Kagan and Yu. V. Kononets, Zh. Eksp. Teor. Fiz. 66, 1692 (1974) [Sov. Phys. JETP 39, 832 (1974)].

${ }^{16} \mathrm{~K}$. Dettmann and M. T. Robinson, Phys. Rev. B 10 , 1 (1974); K. Dettmann, Z. Phys. A 272, 227 (1975).

${ }^{17}$ P. A. Doyle and P. S. Turner, Acta Crystallogr. A 24, 390 (1968).

${ }^{18} \mathrm{E}$. Bonderup, H. Esbensen, J. U. Andersen, and H. E. Schiøtt, Radiat. Eff. 12, 261 (1972). H. Schiøtt, E. Bonderup, J. U. Andersen, and H. Esbensen, in Proceedings of the Fifth International Conference on Atomic Collisions in Solids, Gatlinburg, Tenn., 1973 (Plenum, New York, 1975), Vol. 2, p. 843.

${ }^{19} \mathrm{O} . \mathrm{W}$. Holl and and B. R. Appleton (unpublished).

${ }^{20}$ T. Tomimasu, T. Mikado, and T. Yamazaki, J. Phys. Soc. Jpn. 37, 286 (1974).

${ }^{21}$ H. Esbensen, O. Fich, J. A. Golovchenko, S. Madsen,

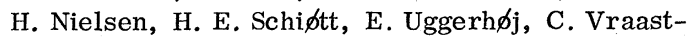
Thomsen, G. Charpak, S. Majewski, G. Odyniec, G. Petersen, F. Sauli, J. P. Ponpon, and P. Siffert, Phys. Lett. B 72, 408 (1978).

${ }^{22} \mathrm{D}$. Gemmell (private communication). 